\title{
Epidemiologic Features and Clinical Subgroups of Anotia/Microtia in Texas
}

\author{
Mark A. Canfield ${ }^{1 *}$ Peter H. Langlois, ${ }^{1}$ Ly M. Nguyen, ${ }^{2}$ and Angela E. Scheuerle ${ }^{1,3}$ \\ ${ }^{1}$ Texas Birth Defects Epidemiology \& Surveillance Branch, Texas Department of State Health Services, Austin, Texas \\ ${ }^{2}$ University of Michigan, Department of Epidemiology, Ann Arbor, Michigan \\ ${ }^{3}$ Tesserae Genetics, Dallas, Texas \\ Received 29 April 2009; Revised 23 July 2009; Accepted 24 July 2009
}

\begin{abstract}
BACKGROUND: Few studies have investigated the epidemiologic features of clinically defined subgroups of anotia/microtia. METHODS: Data on cases of anotia and/or microtia among 1999-2005 deliveries were obtained from the Texas Birth Defects Registry, a population-based active surveillance system. We determined crude and adjusted associations between selected factors and seven clinical subgroups of anotia/ microtia. RESULTS: In total, 742 cases were diagnosed with anotia and/or microtia, corresponding to a prevalence of 2.86 per 10,000 live births. Of those, $45 \%$ had no other major birth defect ("isolated"), $77 \%$ were unilateral, and $22 \%$ bilateral. Anotia alone made up $6 \%$, whereas microtia made up $94 \%$. Birth prevalence was higher with increasing maternal age and among Mexico-born Hispanics. Compared to white mothers, Hispanic mothers were two-to-three times more likely to have infants with all but the syndromic and bilateral groups (adjusted prevalence ratios $[\mathrm{aPRs}]=2.05-2.61$ ). Non-Hispanic blacks had significantly lower risk for total anotia/microtia, and for the isolated, unilateral, and microtia subgroups (aPRs $=0.42-0.64$ ). Less educated mothers were three-to-four times more likely to have children with anotia (aPRs $=2.98$ for less than high school, 3.97 for high school graduates). Males were more likely to be born with total anotia/microtia and with syndromic, unilateral, and microtia subtypes (aPRs $=1.27-1.41)$. CONCLUSIONS: In Texas, most anotia/microtia cases were in the unilateral and microtia groups, and $45 \%$ were isolated. Several clinical subgroups exhibited higher prevalence in males and among older mothers. Relative to whites, blacks were at lower risk and Hispanics (especially Mexico-born mothers) were at higher risk for selected types of anotia/microtia. Birth Defects Research (Part A) 85:905-913, 2009. (C) 2009 Wiley-Liss, Inc.
\end{abstract}

Key words: microtia; anotia; ear; Texas; birth defect; prevalence; epidemiology; risk factor

\section{INTRODUCTION}

Anotia and/or microtia comprise a spectrum of ear birth defects that involve the ear pinna, tragus, lobe, and external auditory canal. Microtia is defined as an abnormality of the ear, varying from a significantly small external ear with minor structural deviations to an external ear with major structural malformations (Carey et al., 2006). Anotia is on the most severe end of the spectrum and is defined as a total absence of the ear and external auditory canal (Carey et al., 2006). Anotia and/or microtia may be unilateral or bilateral.

There have been five other population-based descriptive studies of anotia/microtia, including in three U.S. states: California, Hawaii, and Texas (Mastroiacovo et al., 1995; Harris et al., 1996; Shaw et al., 2004; Forrester and Merz, 2005; Husain et al., 2008). From these five studies, anotia/microtia prevalence ranged from 0.76 per 10,000 births in France (Harris et al., 1996) to
2.77 per 10,000 live births in Texas (Husain et al., 2008), but on average 1.84 per 10,000 births (Harris et al., 1996). The isolated subgroup made up $25 \%$ of all cases

Results from earlier dataset (1999-2001) were presented as a poster at the Annual Meeting of the National Birth Defects Prevention Network, January 30-February 1, 2006, Arlington, VA.

This research was supported in part by a cooperative agreement (\#450/CCU613232) between the Centers for Disease Control and the Texas Center for Birth Defects Research and Prevention, housed at the Texas Department of State Health Services, as well as a contract (\#250-01-00030003(02)) from the Maternal and Child Health Bureau's Graduate Student Internship Program. The contents are solely the responsibility of the authors and do not necessarily represent the official views of their respective organizations.

*Correspondence to: Mark A. Canfield, Ph.D., Texas Birth Defects Epidemiology \& Surveillance Branch, Mailcode 1964, P.O. Box 149347, Austin, TX, 78714-9347. E-mail: mark.canfield@dshs.state.tx.us

Published online 16 September 2009 in Wiley InterScience (www.interscience. wiley.com).

DOI: 10.1002/bdra.20626 
in California (Shaw et al., 2004) and $44 \%$ in Texas (Husain et al., 2008).

With respect to the descriptive epidemiology of anotia and/or microtia, associations have been reported between anotia and/or microtia and high maternal age (Harris et al., 1996; Forrester and Merz, 2005), high maternal parity (Castilla and Orioli, 1986; Mastroiacovo et al., 1995; Harris et al., 1996; Forrester and Merz, 2005), race (Harris et al., 1996; Shaw et al., 2004; Forrester and Merz, 2005; Husain et al., 2008), sex (Harris et al., 1996; Sanchez et al., 1997; Shaw et al., 2004, Forrester and Merz, 2005), low maternal education (Shaw et al., 2004; Husain et al., 2008), prenatal drug exposure (Castilla and Orioli, 1986), advanced paternal age (Castilla and Orioli, 1986), low birth weight (Mastroiacovo et al., 1995; Forrester and Merz, 2005), gestational age (Forrester and Merz, 2005), and maternal diabetes (Mastroiacovo et al., 1995; Correa et al., 2008). Conflicting studies report significant or no significant association with maternal or paternal education (Castilla and Orioli, 1986; Mastroiacovo et al., 1995; Shaw et al., 2004), maternal acute or chronic illness (Castilla and Orioli, 1986), vaginal bleeding (Castilla and Orioli, 1986), residence at the time of delivery (Forrester and Merz, 2005, Husain et al., 2008), and gestational age (Castilla and Orioli, 1986).

The previous Texas study (Husain et al., 2008) examined the association of selected sociodemographic and infant characteristics for anotia/microtia and 13 other birth defects thought to be related to vascular disruption; however, that dataset consisted of isolated cases of anotia and microtia combined and included several years (19961998) during which the Registry was not yet statewide.

The current investigation used more recent statewide data (1999-2005) from the Texas Birth Defects Registry to investigate the relationship between seven demographic features and total anotia/microtia, as well as seven clinically defined subgroups of anotia/microtia in Texas: isolated, syndromic, and multiple anomaly anotia/microtia; unilateral and bilateral anotia/microtia; anotia alone; and microtia.

The size and demographic characteristics of the Texas population provide sufficient power to examine the descriptive epidemiology of anotia/microtia clinical subgroups in a more recent time period. In addition, considering the relatively high prevalence of anotia/microtia in Hispanics, it is compelling to more fully examine its occurrence by region and along the 1255-mile border with Mexico, relative to nonborder areas of Texas.

\section{METHODS}

The Texas Birth Defects Registry (TBDR) is a statewide, population-based registry that uses active surveillance to collect information on cases of birth defects. Basically, trained staff members visit medical facilities on a regular basis to identify cases and collect pertinent data. Facility logs and ICD-9 discharge diagnosis listings are examined for potential cases, for which medical records are requested and reviewed. For records meeting residential and other case inclusion criteria, demographic and diagnostic information are abstracted from medical records and linked with vital records (live birth and fetal death certificates) from the Center for Health Statistics at the Texas Department of State Health Services.
To be included into the Registry database, a case must meet the following criteria. The mother must reside in Texas at the time of delivery. The fetus/infant must have at least one structural birth defect as defined by the registry, and the birth defect must be diagnosed before birth or within one year after delivery. This case definition includes all pregnancy outcomes (live births, spontaneous abortions, and induced terminations of any gestational age) from all regions in Texas. To ensure completeness and accuracy of the abstracted data, extensive and routine quality control procedures are imposed for finding and abstracting cases, eliminating duplicates, assignment of six-digit BPA codes (see below), and case review by three board-certified clinical geneticists. This latter process includes an initial clinical review of selected categories of birth defects, and a second clinical review from a $10 \%$ random sample of all cases. The assignment of cases among the three clinicians is also random.

For this investigation, we used data from the Texas Birth Defects Registry (TBDR) for definitive cases of anotia or microtia delivered from 1999 through 2005. For the purpose of the Registry and after the practice of other birth defect surveillance systems, anotia is described as complete absence of the ear pinna, tragus, and lobe regardless of involvement of the external auditory canal. In this study, anotia was assigned if that term was used in the medical records, or if the ear was described as absent. Microtia for this analysis is described as any significant malformation of the pinna with accompanying stricture or atresia of the external auditory canal. Cases described in the medical record as "microtia" without other definition were included. Stricture or atresia of the external auditory canal with normal ear pinna, as well as the converse (malformation of the pinna without indication of external auditory canal abnormality), were excluded. Also excluded were type I microtia, defined as a minor malformation of the pinna without ear canal anomaly, or an ear described only as "small" without any indication of otherwise abnormal structure.

We extracted cases using British Pediatric Association (BPA) codes of 744.010 (anotia) and 744.210 (microtia). These BPA codes were first modified by the Centers for Disease Control and Prevention (CDC) and later by the Texas Registry. Records for every case in this study underwent an additional level of specialized clinical review to ensure that all cases met the study definition, to assign severity level and laterality, and to categorize the cases as isolated or other. This classification review followed the model of that used by the National Birth Defects Prevention Study (Rasmussen et al., 2003). Seven clinical subgroups for anotia/microtia were further defined: isolated, syndromic, or multiple anomaly anotia/microtia; unilateral or bilateral anotia/microtia; anotia without microtia; and microtia with or without anotia. The isolated versus multiple anomaly categories were distinguished by the absence versus presence of other structural birth defects, but without a syndromic diagnosis. Syndromic cases included those with chromosomal and known genetic etiologies, as well as cases in which, in the opinion of the clinical geneticist, a single gene etiology was likely but not diagnosed.

Data on the following demographic characteristics were taken from vital records, or if missing, from medical records: maternal age (six groups), maternal race/ethnicity (non-Hispanic white, non-Hispanic black, 
Table 1

Distribution and Prevalence ${ }^{\mathrm{a}}$ of Clinical Subtypes of Anotia/Microtia

\begin{tabular}{|c|c|c|c|c|c|c|c|c|c|c|c|c|}
\hline \multirow[b]{2}{*}{ Category/subgroup } & \multicolumn{4}{|c|}{ Total } & \multicolumn{4}{|c|}{ Unilateral } & \multicolumn{4}{|c|}{ Bilateral } \\
\hline & Cases & $\%$ & Prevalence $^{\mathrm{a}}$ & $95 \% \mathrm{CI}^{\mathrm{b}}$ & Cases & $\%$ & Prevalence & $95 \% \mathrm{CI}$ & Cases & $\%$ & Prevalence & $95 \% \mathrm{CI}$ \\
\hline Anotia/microtia (total) & 742 & 100.0 & 2.86 & $2.65-3.07$ & 571 & 100.0 & 2.20 & $2.02-2.38$ & 162 & 100.0 & 0.62 & $0.53-0.72$ \\
\hline Isolated & 336 & 45.3 & 1.30 & $1.16-1.43$ & 319 & 55.9 & 1.23 & $1.09-1.36$ & 17 & 10.5 & 0.07 & $0.04-0.10$ \\
\hline Syndromic $^{c}$ & 121 & 16.3 & 0.47 & $0.38-0.55$ & 52 & 9.1 & 0.20 & $0.15-0.26$ & 65 & 40.1 & 0.25 & $0.19-0.32$ \\
\hline Multiple & 285 & 38.4 & 1.10 & $0.97-1.23$ & 200 & 35.0 & 0.77 & $0.66-0.88$ & 80 & 49.4 & 0.31 & $0.24-0.38$ \\
\hline Anotia alone & 44 & 5.9 & 0.17 & $0.12-0.23$ & 26 & 4.6 & 0.10 & $0.07-0.15$ & 18 & 11.1 & 0.07 & $0.04-0.11$ \\
\hline Isolated & 8 & & 0.03 & $0.01-0.06$ & 8 & & 0.03 & $0.01-0.06$ & 0 & & - & - \\
\hline Syndromic & 14 & & 0.05 & $0.03-0.09$ & 7 & & 0.03 & $0.01-0.06$ & 7 & & 0.03 & $0.01-0.06$ \\
\hline Multiple & 22 & & 0.08 & $0.05-0.13$ & 11 & & 0.04 & $0.02-0.08$ & 11 & & 0.04 & $0.02-0.08$ \\
\hline Microtia (total) ${ }^{\mathrm{d}}$ & 698 & 94.1 & 2.69 & $2.49-2.89$ & 545 & 95.4 & 2.10 & $1.93-2.30$ & 144 & 88.9 & 0.56 & $0.47-0.66$ \\
\hline Isolated & 328 & & 1.26 & $1.13-1.40$ & 311 & & 1.20 & $1.07-1.33$ & 17 & & 0.07 & $0.04-0.10$ \\
\hline Syndromic & 107 & & 0.41 & $0.33-0.49$ & 45 & & 0.17 & $0.13-0.23$ & 58 & & 0.22 & $0.17-0.29$ \\
\hline Multiple & 263 & & 1.01 & $0.89-1.14$ & 189 & & 0.73 & $0.62-0.83$ & 69 & & 0.27 & $0.21-0.34$ \\
\hline
\end{tabular}

${ }^{a}$ Birth prevalence $=$ cases per 10,000 live births; total live births $=2,594,295$.

$\mathrm{b}_{95} \%$ confidence interval for birth prevalence.

'Syndrome cases: 49 chromosome syndromes (including 6 Down syndrome), 34 single gene syndromes (including 19 Treacher Collins), and 38 "other" causes.

${ }^{\mathrm{d}}$ Includes five cases of co-occurring anotia. Laterality missing on four syndromic and five multiple microtia cases.

Hispanic, and other), infant sex (female, male), border county residence (residence at delivery in one of the 14 counties adjacent to Mexico vs. elsewhere), and year of infant delivery (1999-2005). Vital records were used to capture maternal education $(<12,12,>12$ years $)$ and maternal birthplace (U.S., Mexico, other).

Birth prevalence (cases per 10,000 live births) and 95\% confidence intervals were calculated overall and for each subgroup and demographic category of anotia/microtia. Total and subgroup live birth denominators were derived from Texas State Vital Statistics. Next, we examined the association of each subgroup with maternal race/ethnicity, age, education, border county residence, maternal resident birthplace, year of infant delivery, and infant sex, using Poisson regression to perform crude and adjusted analyses. The adjusted analyses included all variables in the model. SAS software version 9.1 was utilized for all analyses.

\section{RESULTS}

There were 2,594,295 live births in Texas from 1999 to 2005. A total of 742 cases in the Texas Birth Defects Registry yielded a birth prevalence rate of 2.86 per 10,000 live births for anotia/microtia (Table 1). Over $97 \%$ of all cases were live born (data not shown). Of the total cases, microtia made up $94.1 \%$ (698 cases) and anotia alone made up $5.9 \%$ (44 cases). Five of the microtia cases had co-occurring anotia. The isolated subgroup made up $45.3 \%(336 / 742)$ of all anotia/microtia cases, but only $18.2 \%(8 / 44)$ of the anotia cases. The unilateral subgroup accounted for $94.9 \%(319 / 336)$ of the isolated cases but only $43.0 \%(52 / 121)$ of the syndromic anotia/microtia cases.

Of the 121 syndromic cases, 49 (40\%) were chromosomal, $34(28 \%)$ were single gene syndromes, and $38(31 \%)$ were in "other/unknown" syndromic categories (Table 1). Of the 49 chromosomal cases, 6 were trisomy 21 (including one partial trisomy), 16 were trisomy
18 (including one mosaic trisomy), and 12 were trisomy 13 (data not shown). The most common single gene syndrome was Treacher Collins, with 19 cases.

The birth prevalence of total anotia/microtia appeared to be higher among male births, older mothers, mothers with lower education, and Hispanics (Table 2). Among Hispanics, higher prevalences were observed for mothers born in Mexico. Among the 11 public health regions of Texas, prevalences were lowest in the northeast and southeast areas (1.91 and 1.51 per 10,000, respectively) and highest in the panhandle and south-central (San Antonio) areas (3.79 and 3.72 per 10,000, respectively) (Fig. 1).

Crude prevalence ratios (cPRs) from the independent association and patterns of selected demographic characteristics with each clinical subgroup are given in Table 3. An increasing age trend was observed for total cases ( $p$ $<0.0001)$, cases that were syndromic $(p<0.0001)$ or had multiple anomalies $(p=0.0004)$, and among cases with bilateral anotia/microtia $(p=0.003)$ or microtia alone $(p$ $<0.0001$ ) (data not shown). In the bilateral subgroup, the oldest mothers $(40+$ years of age) had almost four times the prevalence as that of mothers 25-29 years of age (cPR $=3.84 ; 95 \% \mathrm{CI}=1.81-7.40)$. Relative to non-Hispanic white mothers, Hispanic mothers exhibited significantly elevated prevalence ratios in all subgroups with the exception of syndromic cases. The racial/ethnic disparity was most pronounced in the isolated anotia/microtia group, for which Hispanics had a threefold higher prevalence than non-Hispanic whites $(\mathrm{cPR}=2.91 ; 95 \% \mathrm{CI}=$ 2.24-3.83) and more than a sevenfold higher prevalence than non-Hispanic blacks ( $\mathrm{CPR}=7.28$; data not shown). With the exception of anotia cases, the prevalence ratios were highest among the least educated (vs. most educated) mothers.

Relative to U.S.-born mothers, those born in Mexico were roughly twice as likely to deliver a child with anotia/microtia, with the most pronounced disparity seen for isolated cases $(\mathrm{cPR}=2.58 ; 95 \% \mathrm{CI}=2.06-3.23)$. 
Table 2

Birth Prevalence of Anotia/Microtia by Demographic Characteristics, Texas 1999-2005

\begin{tabular}{|c|c|c|c|c|}
\hline Characteristic & Cases $^{\mathrm{a}}$ & $\%$ & $\begin{array}{c}\text { Birth } \\
\text { prevalence }^{\mathrm{b}}\end{array}$ & $95 \% \mathrm{CI}^{\mathrm{c}}$ \\
\hline Total cases & 742 & 100.0 & 2.86 & $2.65-3.07$ \\
\hline \multicolumn{5}{|l|}{ Maternal age (years) } \\
\hline$<20$ & 93 & 12.5 & 2.48 & $2.01-3.04$ \\
\hline $20-24$ & 194 & 26.1 & 2.64 & $2.27-3.01$ \\
\hline $25-29$ & 182 & 24.5 & 2.64 & $2.25-3.02$ \\
\hline $30-34$ & 173 & 23.3 & 3.33 & $2.83-3.83$ \\
\hline $35-39$ & 82 & 11.1 & 3.60 & $2.86-4.46$ \\
\hline$\geq 40$ & 18 & 2.4 & 3.80 & $2.25-6.01$ \\
\hline \multicolumn{5}{|l|}{ Maternal race/ethnicity } \\
\hline White non-Hispanic & 183 & 24.7 & 1.88 & $1.61-2.15$ \\
\hline Black non-Hispanic & 34 & 4.6 & 1.18 & $0.82-1.66$ \\
\hline Hispanic & 508 & 68.5 & 4.10 & $3.74-4.46$ \\
\hline Border residence & 118 & 15.9 & 3.75 & $3.07-4.42$ \\
\hline Nonborder residence & 390 & 52.6 & 4.22 & $3.80-4.64$ \\
\hline Born in the United States & 211 & 28.4 & 3.43 & $2.97-3.90$ \\
\hline Born in Mexico & 252 & 34.0 & 4.58 & $4.01-5.14$ \\
\hline Other/unknown birthplace & 45 & 6.1 & 6.12 & $4.46-8.19$ \\
\hline Other/unknown race non-Hispanic & 17 & 2.3 & 1.77 & $1.03-2.84$ \\
\hline \multicolumn{5}{|l|}{ Public Health Service region } \\
\hline 1: Panhandle (Lubbock) & 33 & 4.4 & 3.79 & $2.61-5.32$ \\
\hline 2: Abilene/Wichita Falls & 14 & 1.9 & 2.70 & $1.48-4.54$ \\
\hline 3: Dallas/Fort Worth & 209 & 28.2 & 2.99 & $2.59-3.40$ \\
\hline 4: Northeast (Tyler) & 19 & 2.6 & 1.91 & $1.15-2.99$ \\
\hline 5: Southeast (Beaumont) & 8 & 1.1 & 1.15 & $0.50-2.26$ \\
\hline 6: Houston/Galveston & 128 & 17.3 & 2.08 & $1.72-2.44$ \\
\hline 7: Central (Austin/Waco) & 84 & 11.3 & 3.06 & $2.44-3.79$ \\
\hline 8: South Central (San Antonio) & 94 & 12.7 & 3.72 & $3.00-4.55$ \\
\hline 9: West (Midland/Odessa) & 19 & 2.6 & 3.31 & $1.99-5.16$ \\
\hline 10: Far West (El Paso) & 36 & 4.9 & 3.52 & $2.46-4.87$ \\
\hline 11: South (Harlingen/Laredo) & 98 & 13.2 & 3.43 & $2.78-4.18$ \\
\hline \multicolumn{5}{|l|}{ Maternal education } \\
\hline Less than high school & 300 & 40.4 & 3.61 & $3.20-4.01$ \\
\hline High school graduate & 192 & 25.9 & 2.46 & $2.12-2.81$ \\
\hline More than high school & 222 & 29.9 & 2.35 & $2.04-2.66$ \\
\hline Unknown & 28 & 3.8 & - & - \\
\hline \multicolumn{5}{|l|}{ Year of infant birth } \\
\hline 1999 & 99 & 13.3 & 2.84 & $2.30-3.45$ \\
\hline 2000 & 97 & 13.1 & 2.67 & $2.17-3.26$ \\
\hline 2001 & 106 & 14.3 & 2.90 & $2.35-3.46$ \\
\hline 2002 & 106 & 14.3 & 2.85 & $2.30-3.39$ \\
\hline 2003 & 99 & 13.3 & 2.62 & $2.13-3.19$ \\
\hline 2004 & 128 & 17.3 & 3.36 & $2.77-3.94$ \\
\hline 2005 & 107 & 14.4 & 2.78 & $2.25-3.30$ \\
\hline \multicolumn{5}{|l|}{ Infant sex } \\
\hline Male & 421 & 56.7 & 3.17 & $2.87-3.48$ \\
\hline Female & 320 & 43.1 & 2.52 & $2.25-2.80$ \\
\hline
\end{tabular}

${ }^{\mathrm{a}}$ May not add up to total cases if missing value for characteristic.

${ }^{\mathrm{b}}$ Cases per 10,000 live births; total live births $=2,594,295$.

${ }^{\mathrm{c}} \mathrm{95} \%$ confidence intervals.

Residents of the U.S.-Mexico border (vs. nonborder Texas residents) were also more likely to have anotia/microtia, except in the syndromic and bilateral subgroups.

Adjusted prevalence ratios (aPRs) are shown in Table 4, using all available demographic variables except public health region. A significant age trend $(p<0.05)$ remained for all subgroups except "isolated" and anotia (data not shown). Upon adjustment, the oldest mothers (40+ years) had a fourfold higher risk for bilateral anotia/microtia $(\mathrm{aPR}=4.27 ; 95 \% \mathrm{CI}=1.67-9.48)$, relative to the 25-29 age group. A significant racial and ethnic disparity remained upon adjustment. Relative to non-Hispanic whites, Hispanics were more than twofold more likely to have children with every clinical subtype except syndromic and bilateral, and non-Hispanic blacks were significantly less likely to have the isolated, unilateral, and microtia subtypes. Mothers born in Mexico (vs. those born in the U.S.) retained their elevated risk for both "isolated" $(\mathrm{aPR}=1.46 ; 95 \% \mathrm{CI}=1.07-2.00)$ and "syndromic" $(\mathrm{aPR}=1.64 ; 95 \% \mathrm{CI}=1.01-2.69)$ phenotypes. 


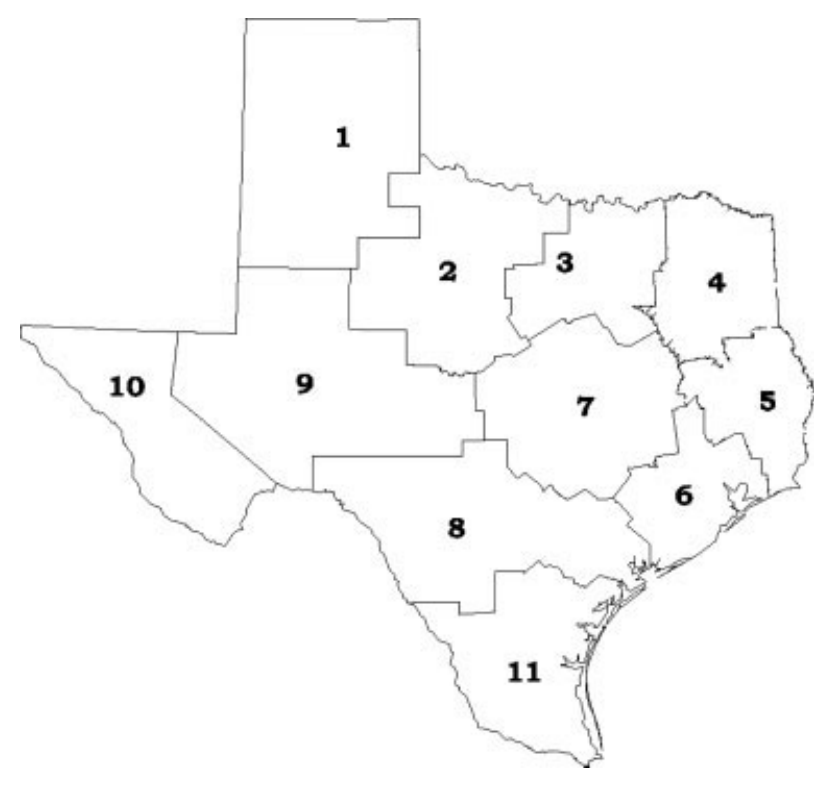

Figure 1. Texas Public Health Service regions. 1: Panhandle (Lubbock/Amarillo); 2: Abilene/Wichita Falls; 3: Dallas/Fort Worth; 4: Northeast (Tyler/Texarkana); 5: Southeast (Beaumont/ Port Arthur); 6: Houston/Galveston; 7: Central (Austin/Waco); 8: South Central (San Antonio); 9: West (Midland/Odessa/San Angelo); 10: Far West (El Paso); 11: South (Harlingen/Laredo/ Corpus Christi).

The increased risk that was suggested for border residents in the crude analyses disappeared upon adjustment. In a similar fashion, the effect of lower education that was observed in the crude analyses for most of the clinical types also disappeared except for anotia alone. The $30-40 \%$ excess risk in males seen in the crude analyses was confirmed for the total cases and for the microtia, syndromic, and unilateral subtypes.

\section{DISCUSSION}

In Texas the birth prevalence of anotia/microtia in 1999-2005 was 2.86 per 10,000 live births, which was comparable to the rate of 2.77 reported in an earlier Texas study (Husain et al., 2008). However, the Texas prevalence was somewhat higher than in reports from earlier population-based studies conducted in Italy, France, Sweden, and California (Mastroiacovo et al., 1995; Harris et al., 1996; Shaw et al., 2004), where prevalence rates ranged between 0.83 and 2.50 per 10,000 births. This difference may have been due to differences among the registries, their inclusion criteria (e.g., whether "chromosomal" cases were included in the numerator and stillbirths in the denominator), and their case ascertainment, as well as some attribute of the population of Texas. With its proximity to Mexico, Texas has a higher proportion of Hispanics. In general, the literature has reported higher rates of anotia/ microtia with the Hispanic and Asian populations and lower rates with the African American and Caucasian populations (Harris et al., 1996; Shaw et al., 2004; Forrester and Merz, 2005; Husain et al., 2008). Hawaii, with an anotia/microtia birth prevalence of 3.79 per 10,000 live births, is the only U.S. state to date to record higher prevalence rates than Texas (Forrester and Merz, 2005). The higher Hawaiian rates may be due to a much larger percentage of persons of Asian descent than in Texas.

Multivariable analyses from the current investigation supported an association between anotia/microtia and maternal race/ethnicity as seen in the literature, as well as maternal age and maternal birthplace, depending on the clinical subtype. Our adjusted Hispanic:white prevalence ratio of 2.6 for the "isolated" subgroup was consistent with the ratio of 3.1 from a previous Texas study of isolated cases (Husain et al., 2008). However, although significantly elevated, these Texas ratios were considerably lower than those reported by the California group (Shaw et al., 2004) for their presumably comparable "isolated" subgroup (4.6 for U.S.-born Hispanics and 6.5 for foreign-born). To our knowledge, our dataset is the first to show markedly lower risk of several types of anotia/ microtia among non-Hispanic blacks, relative to nonHispanic whites. In addition, we are the first to have sufficient power to show increased risk among much older mothers (e.g., bilateral subtype in the $40+$ age group). The California group (Shaw et al., 2004) found a protective effect for teenaged mothers among nonisolated anotia/microtia cases, which we did not replicate. However, we did find a significantly increasing age trend among the nonisolated Texas cases (syndromic and multiple). Our finding of increased prevalence or risk among Mexico-born Hispanics is consistent with findings in California (Shaw et al., 2004).

Dividing anotia/microtia into clinical subgroups provided some interesting and unique results. Of the total cases of external ear defects in our study, $6 \%$ had anotia alone, and $94 \%$ microtia. This finding is consistent with other studies' findings where anotia occurs less commonly than microtia, although some studies have observed a higher proportion of anotia cases than we did, ranging between 13-22\% (Mastroiacovo et al., 1995; Harris et al., 1996; Shaw et al., 2004; Forrester and Merz, 2005; Carey et al., 2006). In the California study, only $2 \%$ of the cases were anotia.

In our dataset, male infants had a significantly increased risk (29-41\%) for the microtia, bilateral, and syndromic subtypes. In the California study, males had a $20-30 \%$ increased risk for isolated and nonisolated (but nonchromosomal) subtypes, but the adjusted relative risk estimates included 1.0 (Shaw et al., 2004). Of all the subgroups we examined, only anotia was highly associated with lower maternal education levels. We believe this to be a unique finding, although we did not further examine this relationship for anotia in the isolated, multiple, and syndromic subcategories, because of small sample size. Although Shaw et al. (2004) did not examine anotia specifically in California, they did find a significantly protective effect of higher education levels for nonisolated anotia/microtia. In the earlier Texas study, Husain et al. (2008) reported a significantly increased risk for isolated anotia/microtia among the least educated mothers ( $<12$ years of education), but we were unable to replicate this finding in our adjusted analysis.

Based on the literature, fewer than $66 \%$ of the total cases of anotia/microtia are considered to be "isolated" 


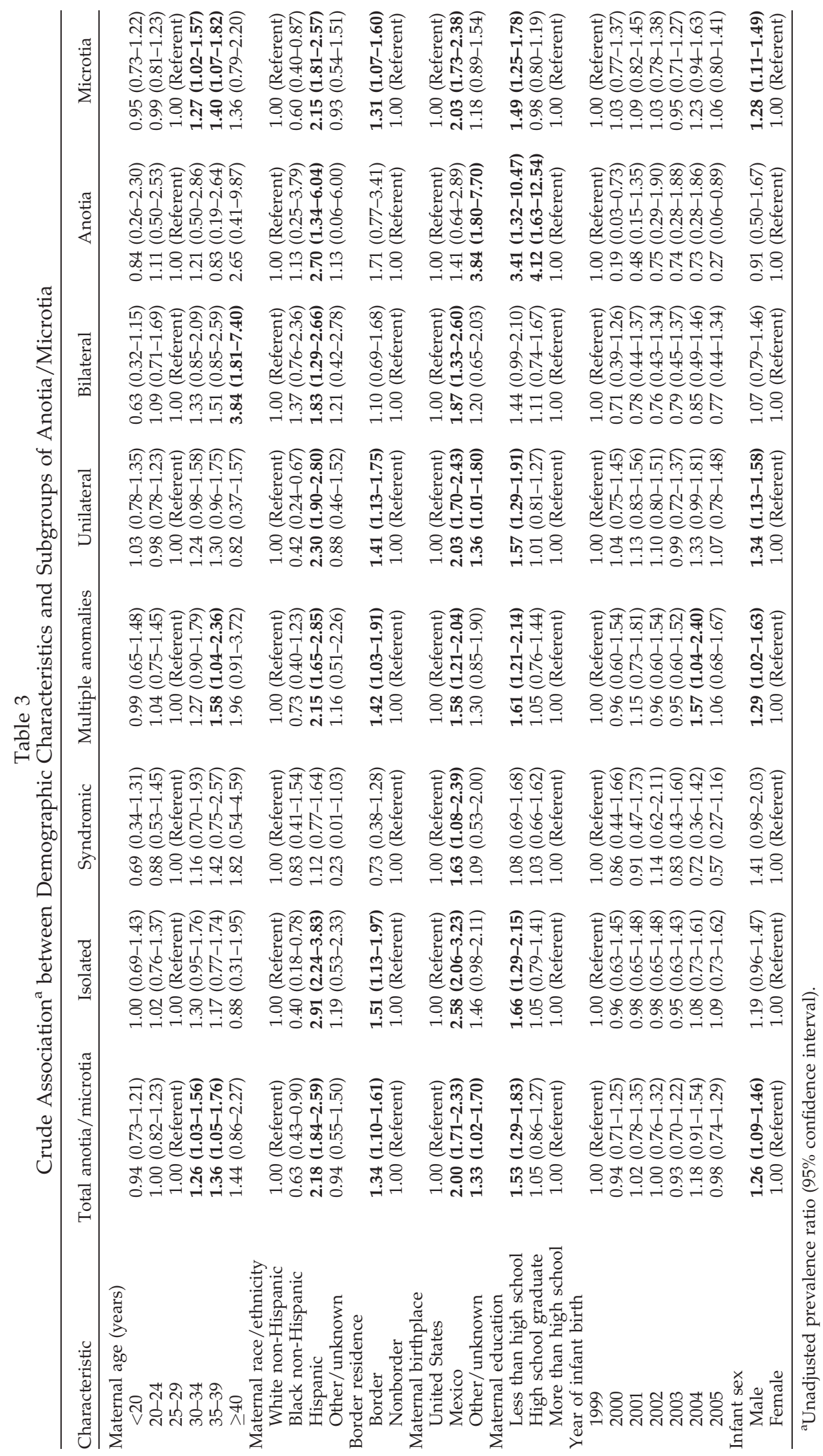




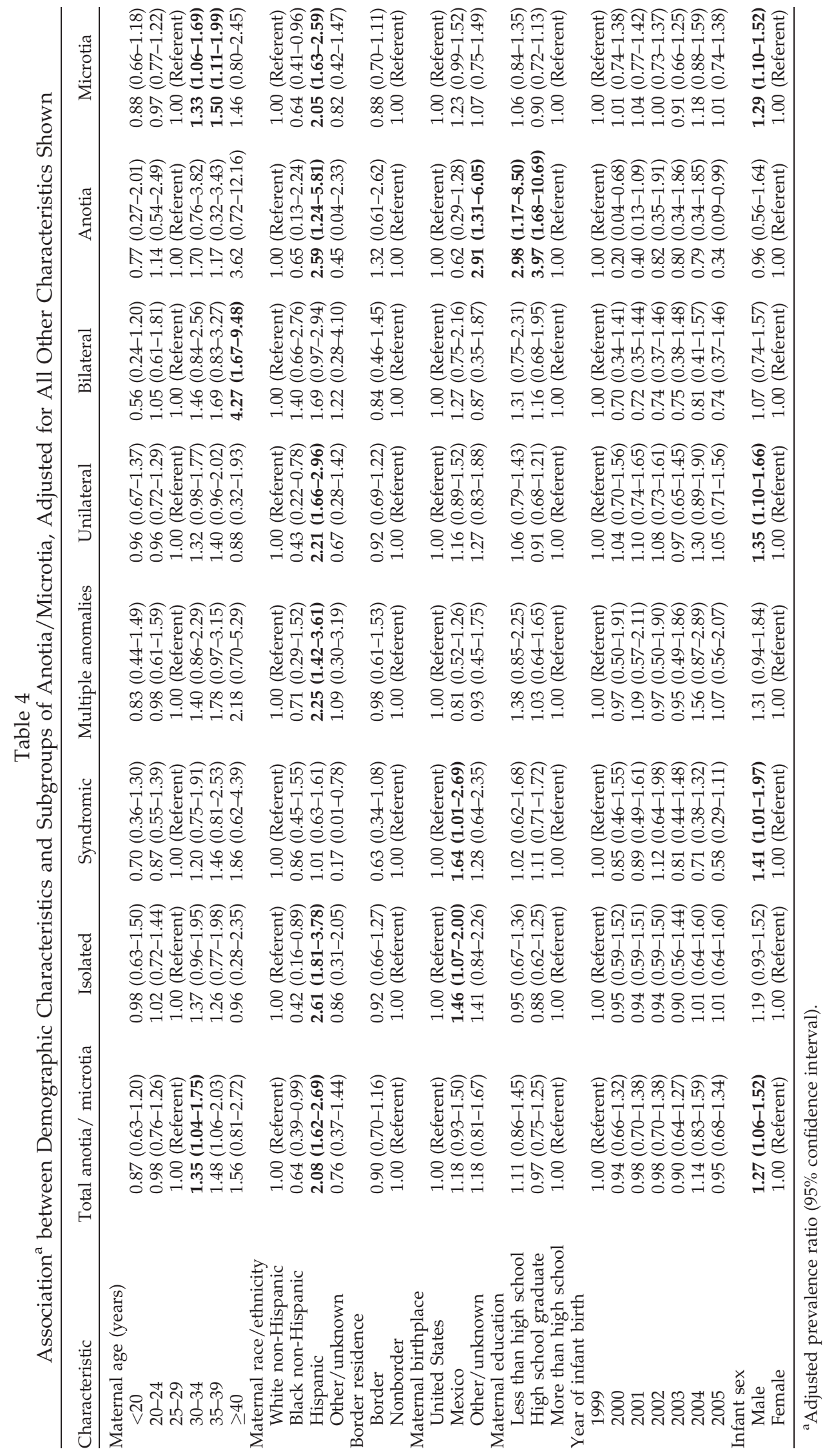

Birth Defects Research (Part A) 85:905-913 (2009) 
(Castilla and Orioli, 1986; Gupta and Patton, 1995; Mastroiacovo et al., 1995; Harris et al., 1996; Sanchez et al., 1997; Shaw et al., 2004; Forrester and Merz, 2005). The isolated subgroup made up $25 \%$ of total cases in California and $45 \%$ in Texas. Texas cases were classified such that an "isolated" case may have minor associated (ex: preauricular pit) or unassociated (ex: sacral dimple) anomalies. This classification is consistent with that used for defect classification in the National Birth Defects Prevention Study (Rasmussen et al., 2003). In our dataset, we observed different patterns of association between the isolated, multiple, and syndromic subgroups (e.g., age, sex, race/ethnicity, maternal birthplace).

In our dataset, $7 \%(49 / 742)$ of the total cases were caused by chromosomal syndromes, of which only six cases were confirmed as Down syndrome $(<1 \%$ of all cases). The corresponding figures for the California study were considerably higher: $14 \%$ for all chromosomal syndromic diagnoses and 5\% for syndromic cases confirmed as Down syndrome (Shaw et al., 2004). This difference might be due to occasional difficulties in retrieving outpatient chromosomal results to make a definitive diagnosis for these types of syndromic cases in Texas.

Of the 742 anotia/microtia cases in this investigation, $77 \%$ were unilateral. This finding was similar to other studies where approximately $80 \%$ or more of the cases were reported as being unilateral (Mastroiacovo et al., 1995; Harris et al., 1996; Sanchez et al., 1997; Shaw et al., 2004; Forrester and Merz, 2005). Bilateral cases made up $22 \%$ of the total Texas cases, whereas they made up $15 \%$ of all cases in Italy (Mastroiacovo et al., 1995). In California, the bilateral group made up $18 \%$ of the total $(5 \%$ of the isolated and $23 \%$ of nonisolated, but nonchromosomal) (Shaw et al., 2004), which was similar to Texas (6\% for the isolated group and $28 \%$ for the comparable "multiple" subgroup). Hispanics and male infants were more likely and non-Hispanic blacks were less likely to have unilateral anotia/microtia. The oldest maternal age group $(40+$ years of age) was highly associated with the bilateral subtype.

The literature exhibited conflicting findings for maternal age. Although some studies appeared to show increasing trends with increasing maternal age (Harris et al., 1996; Shaw et al., 2004), other studies showed no significant trend with changes in maternal age (Mastroiacovo et al., 1995; Forrester and Merz, 2005; Husain et al., 2008). As mentioned above, our adjusted analysis revealed significant age trends for the syndromic and multiple subgroups, and statistically elevated prevalence ratios only for advanced maternal age $(40+$ years of age) and bilateral anotia/microtia. This may be attributed in part to chromosomal aneuploidies. The small but otherwise normally formed ear often found in children with Trisomy 21 is not counted as microtia by the Texas registry. Therefore, the maternal age effect relative to Down syndrome does fully explain the observed association between older maternal age and bilateral microtia in this analysis. The age effect for the smaller bilateral group may be due to other aneuploidies and to single gene disorders with their accompanying paternal age effects.

Only one study reported regional variation of this birth defect. In an earlier Texas study, Husain et al. (2008) observed a significantly increased risk for isolated anotia/microtia among residents of Texas counties bordering
Mexico. We were unable to replicate this finding. We did not see much other regional variation in the occurrence of this defect across Texas, with the exception of relatively low prevalences in east Texas. However, this area of the state contains the highest percentages of nonHispanic blacks, for which prevalence was also low.

This investigation had several limitations that may have led to an underestimation or an overestimation or anotia/microtia cases in Texas. Diagnoses made outside of Texas, diagnoses that changed later than one year after birth, or diagnoses made at non-hospital-based outpatient facilities would not be included in the registry. Furthermore, differing interpretations among staff members and physicians of collected data, coding, and diagnoses could occur and may have particularly affected the proportion of cases that were designated "anotia". In terms of studying race/ethnicity, this investigation was limited to the Caucasian, African American, and Hispanic populations in Texas.

On the other hand, there were several strengths to this study. It drew cases from many live births ( $\mathrm{n}>2.5$ million). Additionally, Texas is home to approximately 7.8 million Hispanics or approximately $18 \%$ of the total 41.3 million Hispanics in the United States (United States Census Bureau, 2005). Not only does Texas share its 1255-mile border with Mexico, it also encompasses three of the top 10 largest cities in the United States. This may be one of the only studies that had enough non-Hispanic black cases to explore the occurrence of various anotia/microtia clinical subtypes in that group. An additional strength relates to the fact that this is the first descriptive study conducted exclusively after the fortification of the U.S. grain supply with folic acid. It is unclear whether folic acid is helpful in preventing external ear defects.

Using data from the Texas Birth Defects Registry, this study found that mothers of Hispanic descent were more likely and non-Hispanic blacks were less likely to deliver a child with anotia/microtia, especially the isolated and microtia subtypes. Males were more likely to have the unilateral and microtia subtypes, and mothers in the oldest age group were at a greater risk for anotia.

\section{ACKNOWLEDGMENTS}

The authors would like to thank the field staff, Amy Case, and Tunu Loponi Ramadhani of the Birth Defects Epidemiology \& Surveillance Branch of the Texas Department of State Health Services. The contents are solely the responsibility of the authors and do not necessarily represent the official views of their respective organizations.

\section{REFERENCES}

Carey JC, Park AH, Muntz HR. 2006. External ear. In: Stevenson ED, Hall JG, Goodman RM, editors. Human malformations and related anomalies. 2nd edition. Oxford: Oxford University Press. pp. 329-335.

Castilla EE, Orioli IM. 1986. Prevalence rates of microtia in South America. Int J Epidemiol 15:364-368.

Correa A, Gilboa SM, Besser LM, Botto LD, Moore CA, Hobbs CA, Cleves MA, Riehle-Colarusso TJ, Waller DK, Reece A, and the National Birth Defects Prevention Study. 2008. Diabetes and structural birth defects. Am J Obstet Gynecol 2008;199:237.e1-e9.

Forrester MB, Merz RD. 2005. Descriptive epidemiology of anotia and microtia, Hawaii, 1986-2002. Congenital Anom 45:119-124.

Gupta A, Patton MA. 1995. Familial microtia with meatal atresia and conductive deafness in five generations. Am J Med Genet 59:238-241. 
Harris J, Kallen B, Robert E. 1996. The epidemiology of anotia and microtia. J Med Genet 33:809-813.

Husain T, Langlois PH, Sever LE, Gambello MJ. 2008. Descriptive epidemiologic features shared by birth defects thought to be related to vascular disruption in Texas, 1996-2002. Birth Defects Res A Clin Mol Teratol 82:435-440.

Mastroiacovo P, Corchia C, Botto LD, et al. 1995. Epidemiology and genetics of microtia-anotia: a registry based study on over one million births. J Med Genet 32:453-457.

Rasmussen SA, Olney RS, Holmes LB, Lin AE, Kepler-Noreuil KM, Moore CA, and the National Birth Defects Prevention Study. 2003.
Guidelines for case classification for the National Birth Defects Prevention Study. Birth Defects Res A Clin Mol Teratol 67:193-201.

Sanchez O, Mendez Gomez E, Guerra D. 1997. Clinico-epidemiologic study of microtia. Invest Clin 38:203-217.

Shaw GM, Carmichael SL, Kaidarova Z, Harris J. 2004. Epidemiologic characteristics of anotia and microtia in California, 1989-1997. Birth Defects Res A Clin Mol Teratol 70:472-475.

United States Census Bureau. 2005. State and county quick facts: 2004 population estimates. Retrieved 21 March 2006 at http://quickfacts. census.gov/qfd/states/html 